\title{
Another look into decomposition results
}

\author{
Jevgenijs Ivanovs • Offer Kella
}

Received: 3 July 2012 / Revised: 11 December 2012 / Published online: 5 March 2013

(C) Springer Science+Business Media New York 2013

\begin{abstract}
In this note, we identify a simple setup from which one may easily infer various decomposition results for queues with interruptions as well as càdlàg processes with certain secondary jump inputs. Special cases are processes with stationary or stationary and independent increments. In the Lévy process case, the decomposition holds not only in the limit but also at independent exponential times, due to the Wiener-Hopf decomposition. A similar statement holds regarding the GI/GI/1 setting with multiple vacations.
\end{abstract}

Keywords Lévy process - Random walk - Reflection - Secondary jump input . G/G/1 queue $\cdot$ Server vacations $\cdot$ Wiener-Hopf factorization

Mathematics Subject Classification Primary: 60K25 - Secondary: 60G50 - 60G51

\section{Introduction}

The motivation for this study is as follows. Consider a process that, whenever it is nonnegative, is uninterrupted, and upon its attempt to become negative an external positive jump (or jumps) is added to prevent it from doing so. Such a process with a secondary jump input can model fluid queues or storage processes, where a server

\footnotetext{
J. Ivanovs ( $\square)$

Department of Actuarial Sciences, University of Lausanne,

Bâtiment Extranef, 1015 Lausanne, Switzerland

e-mail: jevgenijs.ivanovs@unil.ch

O. Kella

Department of Statistics, The Hebrew University of Jerusalem, Mount Scopus, 91905 Jerusalem, Israel e-mail: Offer.Kella@huji.ac.il
} 
becomes unavailable for certain periods of time due to the insertion of external work. It can also serve as a weak limit of certain queues with server vacations; See, for example $[10,13]$. Alternatively, external jumps may represent orders made and immediately received in a warehouse system. Yet another example comes from risk theory, where an external jump input may be viewed as injection of capital by investors in order to prevent a company going bankrupt.

Similarly, one can consider a discrete-time analog, i.e., a random walk with a secondary jump input, which may represent a G/G/1 queue with server vacations or interruptions. Notably, certain stochastic decomposition results hold for various models of this type, see for example [6], for an $\mathrm{M} / \mathrm{G} / 1$ queue with server vacations, and $[3-5,7,9,12,14]$ for a general case of a $G / G / 1$ queue. In the continuous time case, however, such decomposition results are only known when the driving process is Lévy and moreover has no negative jumps; see [11]. In the latter model the stationary workload can be decomposed into the sum of two independent random variables: one corresponding to the stationary workload in the usual Lévy-driven queue without secondary jumps (the reflected process) and the other to the stationary residual lifetime distribution associated with the renewal process defined by the secondary jumps alone.

The purpose of this note is threefold. The first is to identify a simple truth the decomposition lemma: Lemma 1) which implies the above decompositions in both the discrete and continuous time cases. The second is to apply this decomposition Lemma to various continuous and discrete time queues or storage models with vacations or secondary jump inputs in order to establish a recognized decomposition result for these processes. The third goal is to identify a decomposition for the Lévy case (with possibly two-sided jumps) at independent exponentially distributed times, as well as the random walk case at independent geometrically distributed times. This is due to the Wiener-Hopf decomposition and extends earlier results for Lévy processes with no negative jumps.

It turns out that the limiting decomposition results are not a consequence of the special assumptions on the underlying driving process. These assumptions are important, but usually only for the purpose of giving an explicit representation for the distribution of a certain part of the decomposition. The main idea that allows us to use the decomposition Lemma is the observation that one may represent the difference between the process with vacations (or secondary jump input) and the reflected process as the residual lifetime process associated with the vacations evaluated at the negative of the running infimum of the driving process (to be made precise later). It turned out that a seed to this idea was actually planted long before. Namely, one may interpret the observations made by [3] as a special case of this setup for the GI/GI/1 queue with vacations when one looks at arrival instants, because then the negative of the running minimum is in fact a sum of completed idle times (which is what appears there). While trying to understand the proof of the decomposition result as given there, we detected an error, even though the end result is correct as was also observe earlier (see [7]). In the concluding section of this note we give a simple counter example to the assumption made in [3].

Throughout this note we use the following notation: $a \vee b=\max (a, b), a \wedge b=$ $\min (a, b), a^{+}=a \vee 0$ and $a^{-}=a \wedge 0$. 


\section{A decomposition lemma}

The following is what we call the decomposition lemma. Despite its simplicity, it turns out to be the essential reason for decomposition results discussed in this paper.

Lemma 1 Let $\left(X_{t}\right)_{t \geq 0}$ and $\left(Y_{t}, Z_{t}\right)_{t \geq 0}$ be stochastic processes where

- $\left(X_{t}\right)_{t \geq 0}$ is right (or left) continuous a.s.,

- $X_{s}$ is independent of $\left(Y_{t}, Z_{t}\right)$ for each $s, t \geq 0$,

- $Z_{t} \geq 0$ a.s.

Assume that

$$
X_{t} \stackrel{d}{\rightarrow} X, \quad Y_{t} \stackrel{d}{\rightarrow} Y, \quad Z_{t} \stackrel{p}{\rightarrow} \infty, \quad \text { as } t \rightarrow \infty
$$

Then $\left(X_{Z_{t}}, Y_{t}\right) \stackrel{d}{\rightarrow}(X, Y)$ where $X$ and $Y$ are independent.

It may be helpful to see the application of this lemma given in Sect. 3 before reading through the rest of this section. The proof of the decomposition lemma relies on the following fact.

Lemma 2 On a given probability space $(\Omega, \mathcal{F}, P)$ let $\mathcal{F}_{0} \subset \mathcal{F}$ be a sigma-field and $U=\left\{U_{s} \mid s \geq 0\right\}$ be an a.s. bounded right continuous stochastic process such that $U_{s}$ is independent of $\mathcal{F}_{0}$ for every $s \geq 0$. If $V$ is a nonnegative $\mathcal{F}_{0}$-measurable random variable then

$$
E\left[U_{V} \mid \mathcal{F}_{0}\right]=u(V),
$$

where $u(s)=E U_{s}$.

Proof Letting $\tau_{n}(s)=(\lfloor n s\rfloor+1) / n$ we have $\tau_{n}(s) \downarrow s$ as $n \rightarrow \infty$. Hence by right continuity $U_{\tau_{n}(s)} \rightarrow U_{s}$ and since by bounded convergence $u(s)$ is also right continuous, then also $u\left(\tau_{n}(s)\right) \rightarrow u(s)$ as $n \rightarrow \infty$. Now, for every $A \in \mathcal{F}_{0}$ we have

$$
\begin{aligned}
E U_{\tau_{n}(V)} 1_{A} & =E \sum_{k=1}^{\infty} U_{k / n} 1_{\left\{V \in\left[\frac{k-1}{n}, \frac{k}{n}\right)\right\}} 1_{A} \\
& =\sum_{k=1}^{\infty} u(k / n) E 1_{\left\{V \in\left[\frac{k-1}{n}, \frac{k}{n}\right)\right\}} 1_{A}=E u\left(\tau_{n}(V)\right) 1_{A},
\end{aligned}
$$

where the second step follows by Fubini's theorem and the independence of $U_{k / n}$ from $\mathcal{F}_{0}$. By bounded convergence it follows that

$$
E U_{V} 1_{A}=E u(V) 1_{A}
$$

and, since $u(V) \in \mathcal{F}_{0}$ (by right continuity $u$ is Borel), we are done. 
Proof of Lemma 1 Let $f, g$ be bounded and continuous and denote $h(t)=E f\left(X_{t}\right)$. Then

$$
h(t)=E f\left(X_{t}\right) \rightarrow c \equiv E f(X), \text { as } t \rightarrow \infty
$$

and thus $h\left(Z_{t}\right) \stackrel{p}{\rightarrow} c$. Therefore (Slutsky's Theorem or the "converging together lemma", see [8, p. 318]),

$$
\left(h\left(Z_{t}\right), Y_{t}\right) \stackrel{d}{\rightarrow}(c, Y) .
$$

By Lemma 2 we have $E\left[f\left(X_{Z_{t}}\right) \mid \sigma\left(Y_{t}, Z_{t}\right)\right]=h\left(Z_{t}\right)$ and so

$$
E f\left(X_{Z_{t}}\right) g\left(Y_{t}\right)=E h\left(Z_{t}\right) g\left(Y_{t}\right) \rightarrow c E g(Y)=E f(X) E g(Y),
$$

where convergence follows by taking a bounded and continuous $\phi(\xi, \eta) \equiv(\xi \wedge$ $a \vee(-a)) g(\eta)$ for sufficiently large $a$, so that $E h\left(Z_{t}\right) g\left(Y_{t}\right)=E \phi\left(h\left(Z_{t}\right), Y_{t}\right)$ and $c E g(Y)=E \phi(c, Y)$, which is possible, because $h$ is bounded. Taking $f(\xi)=e^{i \alpha \xi}$ and $g(\eta)=e^{i \beta \eta}$ (or sines and cosines if one insists on real valued functions) implies that in fact $\left(X_{Z_{t}}, Y_{t}\right)$ converges in distribution to $(X, Y)$, where the latter are independent. Finally, if $X_{t}$ is left continuous then a corresponding analogue of Lemma 2 can be used.

Remark 1 We make the following observations:

1. If $X_{t}$ is indexed by $\mathbb{R}$ instead of $[0, \infty)$ then the assumption that $Z_{t} \geq 0$ a.s. is not needed. Alternatively, one may take for example $X_{Z_{t}^{+}}$instead of $X_{Z_{t}}$.

2. Although in the statement of the lemma $X_{t}$ and $Y_{t}$ are one dimensional, in the proof this is not required and thus the decomposition lemma is valid for the multivariate case or even the case where $X_{t}$ and $Y_{t}$ take their values in (possibly different) metric spaces.

3. Clearly, the decomposition lemma holds when $\left(Y_{t}, Z_{t}\right)$ is a discrete time process.

4. When $Z_{t}$ is a nondecreasing process then $Z_{t} \stackrel{p}{\rightarrow} \infty$ if and only if $Z_{t} \rightarrow \infty$ almost surely.

5. Only marginal independence of $X_{s}$ and $\left(Y_{t}, Z_{t}\right)$ is required. Moreover, the assumption of right (or left) continuity of $X_{t}$ can be relaxed or even removed, but at a price of a stronger independence assumption. In what follows this kind of generalization is not needed and we prefer the weaker independence assumption.

\section{Decomposition in stochastic storage processes with secondary jump inputs}

Let $X=\left\{X_{t} \mid t \geq 0\right\}$ be a càdlàg process and let $V_{1}, V_{2}, \ldots$ be i.i.d. positive random variables that are independent of $X$. We let

$$
W_{t}^{v}=X_{t}+\sum_{i=1}^{N^{v}(t)} V_{i}
$$




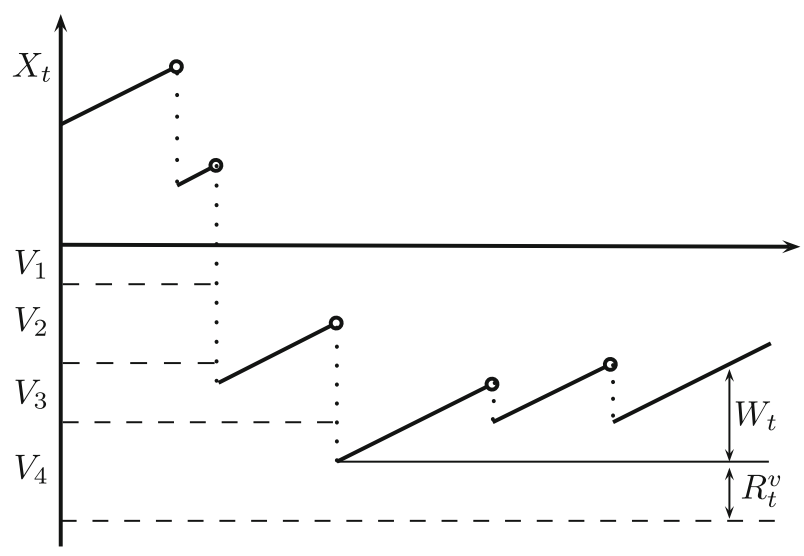

Fig. 1 A schematic sample path of $X$

where $N^{v}(t)$ is the minimal process for which the right hand side is nonnegative for all $t \geq 0$. That is the process $W^{v}$ evolves as the process $X$, but whenever it attempts to become negative, then just enough $V_{i}$ 's (one or more) are instantly added in order to make it nonnegative. Alternatively, one can require that $W^{v}$ be strictly positive instead of nonnegative. To set up some standard renewal process notation, let $S_{n}=\sum_{i=1}^{n} V_{i}, N(t)=\inf \left\{n \mid S_{n}>t\right\}$ and the residual life time process is $R_{t}=S_{N(t)}-t$.

The minimal nonnegative nondecreasing process $Y_{t}$ such that $X_{t}+Y_{t} \geq 0$ for all $t \geq 0$ is $I_{t} \vee 0$, where $I_{t}=-\inf _{0 \leq s \leq t} X_{s}$. Although this is well known, see for example [1, Prop. IX.2.2] for a closely related statement, we recall that this follows from the fact that $0 \leq X_{s}+Y_{s} \leq X_{s}+Y_{t}$ for every $0 \leq s \leq t$ and thus $Y_{t} \geq-X_{s}$ for every $0 \leq s \leq t$. Thus, together with the assumption that $Y_{t} \geq 0$, necessarily $Y_{t} \geq\left(I_{t} \vee 0\right)$. We denote this reflected (or regulated) process by $W_{t}$, i.e.,

$$
W_{t}=X_{t}+I_{t} \vee 0
$$

It turns out that there is a simple relation between the sample paths of the processes $W^{v}$ and $W$, which can be easily seen from Fig. 1 .

We stack the jumps $V_{n}$ one upon another below the level 0 , creating a renewal process (running downwards). Then the value of the process $W^{v}$ can be read off as the distance between the path of $X$ and the closest dashed line from below when $I_{t} \geq 0$. In other words,

$$
W_{t}^{v}=W_{t}+1_{\left\{I_{t} \geq 0\right\}} R_{t}^{v}=W_{t}+1_{\left\{I_{t} \geq 0\right\}} R_{I_{t}},
$$

where $R_{t}^{v}$ is the residual lifetime associated with our renewal process at 'time' $I_{t}$. One should note that representation (4) may not hold when $I_{t}$ coincides with a renewal epoch (irrespective if $R_{t}$ is taken right or left continuous). To see this, assume in addition that $X_{t}=-I_{t}$ and hence $W_{t}=0$. But then $W_{t}^{v}$ can be either $V_{i}$ or 0 , where the first case occurs when $I_{s}>I_{t}$ for all $s>t$ and the second otherwise. Importantly, 
this problem is not essential for the decomposition result as will be shown in the following.

Of course, one can use purely algebraic manipulations to derive representation (4). On the event $I_{t} \geq 0$, by minimality we have $S_{N^{v}(t)-1} \leq I_{t} \leq S_{N^{v}(t)}$. Assuming that these inequalities are strict ( $I_{t}$ does not coincide with a renewal epoch) we get $N\left(I_{t}\right)=N^{v}(t)$ and hence $S_{N^{v}(t)}-I_{t}=R_{I_{t}}$, therefore $W_{t}^{v}=X_{t}+1_{\left\{I_{t} \geq 0\right\}}\left(R_{I_{t}}+I_{t}\right)$ and we are done.

Theorem 1 Assume that $\left\{X_{t} \mid t \geq 0\right\}$ and $\left\{V_{n} \mid n \geq 0\right\}$ are independent, $\liminf _{t \rightarrow \infty} X_{t}=$ $-\infty$ in probability, $W_{t} \stackrel{d}{\rightarrow} W_{\infty}$ as $t \rightarrow \infty$ and $\left\{V_{n} \mid n \geq 1\right\}$ are i.i.d., distributed like some positive $V$ having a nonarithmetic distribution with $E V<\infty$. Then

$$
W_{t}^{v} \stackrel{d}{\rightarrow} W_{\infty}+V_{e}
$$

where $W_{\infty}$ and $V_{e}$ are independent and $P\left(V_{e} \in d x\right)=\frac{P(V>x)}{E V} d x$ (stationary residual life density).

Proof Observe that the process $R$ is right continuous and is independent of the process $(W, I)$. In particular $R_{s}$ is independent of $\left(W_{t}, I_{t}\right)$ for every $s, t \geq 0$. The decomposition lemma (Lemma 1) implies that $\left(1_{\left\{I_{t} \geq 0\right\}} R_{I_{t}}, W_{t}\right)$ converges to the pair $\left(V_{e}, W_{\infty}\right)$, where $V_{e}$ and $W_{\infty}$ are independent, and hence $W_{t}+1_{\left\{I_{t} \geq 0\right\}} R_{I_{t}} \stackrel{d}{\rightarrow} W_{\infty}+V_{e}$. The representation (4) would complete the proof if not the above mentioned problem that it may not hold when $I_{t}$ coincides with a renewal epoch. This is immediately overcome by the fact that $P\left(\exists n: I_{t}=S_{n}\right) \rightarrow 0$ as $t \rightarrow \infty$. The latter follows from $P\left(\exists n: t=S_{n}\right) \rightarrow 0$, see for example [1, Thm. V.4.3], and the independence between $I_{t}$ and the process $\left(S_{n}\right)_{n \geq 0}$. That is, simply write

$$
P\left(\exists n: I_{t}=S_{n}\right) \leq \mathbb{P}\left(I_{t}<T\right)+\int_{T}^{\infty} P\left(\exists n: s=S_{n}\right) P\left(I_{t} \in d s\right)
$$

and argue that the right side is arbitrary small for large enough $t$.

In Theorem 1 , it is assumed that $V$ has a nonarithmetic distribution. This assumption cannot be removed in general as is evident from a simple example, where $X_{t}=-t$, so that $W_{t}=0$ and $W_{t}^{v}=R_{t}$. Nevertheless, there are various ways to get around this restriction. For example, one can assume that the renewal process is stationary, i.e., take $V_{1}$ to be distributed as $V_{e}$.

Remark 2 Once this structure is understood it is clear that various generalizations are possible without change in the final decomposition outcome.

1. Instead of $\left\{V_{n} \mid n \geq 0\right\}$ i.i.d., assume that $\left\{V_{n} \mid n \geq k\right\}$ are i.i.d. for some $k \geq 2$ and are independent of $\left(V_{1}, \ldots, V_{k-1}\right)$, where the latter can have any joint distribution.

2. Instead of $W_{t}=X_{t}+I_{t} \vee 0$ and $1_{\left\{I_{t} \geq 0\right\}} R_{I_{t}}$, one may consider $X_{t}+Y_{t}$ and $R_{Y_{t}}$ for some nonnegative nondecreasing $\left\{Y_{t} \mid t \geq 0\right\}$ for which $X_{t}+Y_{t} \geq 0$ for all $t \geq 0$. Here, $N^{v}(t)$ is the minimal process for which $W_{t}^{v}$ is bounded below by $X_{t}+Y_{t}$. 
3. Instead of $R_{S}$ one may take any other process which is independent of $X$ and thus of $(I, W)$. One example of such a process is as follows. Letting $N(s)=\inf \left\{n \mid V_{n}>s\right\}$ take $R_{S}=\left(V_{N(s)}-s\right)$. In this case,

$$
P\left(R_{s}>u\right)=\frac{P(V>s+u)}{P(V>s)}
$$

so that

$$
P\left(R_{S}>u+v\right)=P\left(R_{S}>u\right) P\left(R_{s+u}>v\right) .
$$

$R_{S}$ does not always converge in distribution as $s \rightarrow \infty$, but when it does and the limit is nondegenerate, then the limiting distribution must be exponential. This happens when $P(V>\log v)=P\left(e^{V}>v\right)$ is regularly varying.

Another example is when $Y$ is some Lévy process idependent of $X$ satisfying $E\left|Y_{t}\right|<\infty$ and $E Y_{t}>0$ and for $s \geq 0, T_{s}=\inf \left\{t \mid Y_{t}>s\right\}$. Then we set $R_{s}=\left(Y_{T_{s}}-s\right)$. Convergence in distribution for this case is discussed in [2].

4. The continuous time setup given here may be replaced by a discrete time version without change. That is $\left\{X_{n} \mid n \geq 0\right\}$ instead of $\left\{X_{t} \mid t \geq 0\right\}$ and $I_{n}=-\min \left(X_{0}, \ldots, X_{n}\right)$ instead of $I_{t}$. This implies the well known decomposition results for the waiting time (in the queue) in a G/G/1 queue with multiple server vacations. Note that all that is required here is to know that the waiting time without the vacations converges in distribution, which may hold for various setups in particular when the service and inter-arrival pairs are not i.i.d.

\section{The case where $X$ has stationary increments}

Assume that $X=\left(X_{t}\right)_{t \in \mathbb{R}}$ is a càdlàg process having stationary increments. That is, for any $s$, the distribution of the process

$$
X^{s}=\left(X_{s+t}-X_{s}\right)_{t \in \mathbb{R}}
$$

is independent of $s$. In addition assume that

$$
\liminf _{t \rightarrow \infty} X_{t}=-\infty \text { and } \inf _{t \leq 0} X_{t}>-\infty
$$

the first in probability and the second almost surely. For example if there are enough conditions (e.g., ergodicity, $E \sup _{0 \leq s \leq t}\left|X_{s}\right|<\infty$ for some $0<t<\infty$ and $E X_{1}<0$ ) that insure that $X_{t} / t \rightarrow c$ a.s. for some $c<0$, both as $t \rightarrow \infty$ as well as $t \rightarrow-\infty$ then the conditions of (6) are met. A special case is when $X$ also has independent increments, that is, $X$ is a Lévy process and then it suffices to assume that $E X_{1}^{+}<\infty$ and that $E X_{1}<0$. Alternatively, we could have also started with a one sided process $\left(X_{t}\right)_{t \geq 0}$ having stationary increments and then make the usual (unique) two sided extension. The following ideas marginally generalize ideas which are not new (e.g., 
[1, Sect. IX.2]), but since they take up just a few lines we found it worthwhile to include them for ease of reference and in order to make the paper more self contained.

Noting that $X_{t}=X_{t}^{0}+X_{0}$, for $t \geq 0$ define

$$
\begin{array}{cc}
I_{t}^{0}=-\inf _{0 \leq s \leq t} X_{s}^{0}, & W_{t}^{0}=X_{t}^{0}+I_{t}^{0}, \\
I_{t}=-\inf _{0 \leq s \leq t} X_{s}^{-}=\left(I_{t}^{0}-X_{0}\right)^{+}, W_{t}=X_{t}+I_{t}=X_{t}^{0}+I_{t}^{0} \vee X_{0} .
\end{array}
$$

A standard Loynes' argument for this case is

$$
W_{t}^{0}=X_{t}^{0}+I_{t}^{0}=\sup _{0 \leq s \leq t}\left(X_{t}-X_{s}\right)=\sup _{0 \leq s \leq t}\left(X_{t}-X_{t-s}\right)=-\inf _{0 \leq s \leq t} X_{-s}^{t},
$$

hence $W_{t}^{0}$ and $M_{t}=-\inf _{-t \leq s \leq 0} X_{s}^{0}$ are identically distributed. So, $W_{t}^{0}$ is stochastically increasing and converges in distribution to that of

$$
W_{\infty} \sim M=-\inf _{s \leq 0} X_{s}^{0}
$$

By our assumptions $M<\infty$ a.s. and $I_{t} \rightarrow \infty$ in probability. Moreover,

$$
W_{t}-W_{t}^{0}=I_{t}^{0} \vee X_{0}-I_{t}^{0}=\left(X_{0}-I_{t}^{0}\right)^{+}
$$

which vanishes in probability since $I_{t}^{0} \geq I_{t} \rightarrow \infty$, and thus $W_{t} \stackrel{d}{\rightarrow} W_{\infty}$ as well. Therefore, if in addition to the above $\left\{V_{n} \mid n \geq 0\right\}$ is independent of $\left\{X_{t} \mid t \in \mathbb{R}\right\}$ and satisfies the requirements of Theorem 1 , then the decomposition result of Theorem 1 holds.

In particular, for the discrete time version of the setup of this section one obtains the decomposition results for waiting times in the G/G/1 queues with stationary structure and multiple i.i.d. vacations with somewhat more general assumptions than considered in the literature. Of course, this also implies the decomposition results for the $G I / G I / 1$ queue.

\subsection{The Lévy case and decomposition at exponential times}

As was mentioned earlier, when $X$ is a Lévy process satisfying $E\left|X^{+}\right|<\infty$ and $E X_{1}<0$, then the conditions of the previous section are satisfied and thus the decomposition holds. This generalizes the known results for the Lévy case with no negative jumps. Of course, in the latter case, the Laplace-Stieltjes transform of the stationary distribution associated with the reflected Lévy process has an explicit form, which can be used to establish decomposition. In general the transform may not be known explicitly, but nevertheless the decomposition is still valid.

Assume that $X_{0}=0$ and take an exponential random variable $T$ independent of everything else. It is well known that $W_{T}$ and $I_{T}$ are independent. This fact is sometimes called "splitting at the minimum" and is linked to the Wiener-Hopf factorization. In this setting it is more convenient to take the left continuous version of the residual life process $R_{t}$ with $R_{0}=0$. Then $W_{t}^{v}=W_{t}+R_{I_{t}}$ apart from countably many $t$ (these 
are the times when $I_{t}$ coincides with a renewal epoch and immediately surpasses it). Hence

$$
W_{T}^{v}=W_{T}+R_{I_{T}} \text { a.s., }
$$

where the summands are independent.

Remark 3 Equation (8) suggests the decomposition for the limiting distribution of $W_{t}^{v}$ : substitute $T$ with another exponential variable $a T$ and let $a \rightarrow \infty$. There is, however, a "small" detail to be filled in this argument. One has to show that $W_{t}^{v}$ converges in distribution (it only shows that it converges along some special sequence of random times). It seems that covering this gap would not be any easier than via a result like Lemma 1.

An identical argument is valid for the case where continuous time is replaced by discrete time. That is, if $X_{n}=\sum_{i=1}^{n} Y_{i}, Y_{i}$ are i.i.d. and $T$ has a geometric distribution, then $W_{T}$ and $I_{T}$ are independent. Furthermore, in the discrete time setup there are no problems with the representation, i.e. $W_{n}^{v}=W_{n}+R_{I_{n}}$ holds for each $n$. This implies that for a GI/GI/1 queue with multiple i.i.d. vacations, the decomposition holds at any independent geometric time.

\section{A counter example to an independence belief}

A key observation was suggested in [3], see Lemma 1(c) and the comment preceding Lemma 2, according to which in a GI/GI/1 queue the waiting time $W_{n}$ of the $n$th customer is independent of the preceding idle times (and hence of their sum $I_{n}$ ) given the index $N_{n}^{B}=k$ of the busy cycle in which the $n$th customer has arrived. This is further used to claim conditional independence of $W_{n}$ and $R_{n}$ (in our notation). It is easy to believe this and move on, although no actual proof was given. While attempting to understand it a bit better, we devised the following example (among many possible ones).

Assume that the queue is modeled by a random walk with increments being 1 or -2 with equal probability $1 / 2$ (this is the distribution of the preceding service time minus interarrival time). Consider the second arriving customer given that the 0th customer arrives to an empty queue at time 0 , and so $X_{0}=0$. There are four cases to consider, each occurs with probability $1 / 4$, which are given in the following table.

\begin{tabular}{rrrrr}
\hline$X_{1}$ & $X_{2}$ & $W_{2}$ & $I_{2}$ & $N_{2}^{B}$ \\
\hline 1 & 2 & 2 & 0 & 0 \\
-2 & -4 & 0 & 4 & 2 \\
1 & -1 & 0 & 1 & 1 \\
-2 & -1 & 1 & 2 & 1 \\
\hline
\end{tabular}


From this, it is evident that on $\left\{N_{2}^{B}=1\right\}$ the pair $\left(W_{2}, I_{2}\right)$ takes values $(0,1)$ and $(1,2)$ with probability $1 / 2$ for each, which implies that $W_{2}$ and $I_{2}$ are not conditionally independent given $N_{2}^{B}=1$.

Similarly, one can consider the vacation model with $V_{n}=3$ (deterministic). Now, on the event $\left\{N_{2}^{B}=1\right\}$ the pair $\left(W_{2}, R_{2}^{v}\right)$ takes values $(0,2)$ and $(1,1)$ with equal probabilities; here $W_{2}$ denotes the waiting time in the original queue. So again $W_{2}$ and $R_{2}^{v}$ are not conditionally independent given $N_{2}^{B}=1$. This contradicts the observation following Eq. (3.4) in [3].

Acknowledgments Jevgenijs Ivanovs study was supported by the Swiss National Science Foundation Project 200021-124635/1, and Offer Kella study was supported in part by Grant No. 434/09 from the Israel Science Foundation and the Vigevani Chair in Statistics.

\section{References}

1. Asmussen, S.: Applied Probability and Queues (Applications of Mathematics (New York)), 2nd edn, p. 51. Springer, New York (2003)

2. Doney, R.A., Kyprianou, A.E.: Overshoots and undershoots of Lévy processes. Ann. Appl. Probab. 16, 91-106 (2006)

3. Doshi, B.T.: A note on stochastic decomposition in a GI/G/1 queue with vacations or set-up times. J. Appl. Probab. 22, 419-428 (1985)

4. Doshi, B.T.: Queueing systems with vacations: a survey. Queueing Syst. 1, 29-66 (1986)

5. Doshi, B.T.: Generalization of the stochastic decomposition result for single server queues with vacations. Stoch. Models 6, 307-333 (1990)

6. Fuhrmann, S.W., Cooper, R.B.: Stochastic decompositions in the M/G/1 queue with generalized vacations. Oper. Res. 33(5), 1117-1129 (1985)

7. Gelenbee, E., Iasnogorodski, R.: A queue with server of walking type (autonomous service). Ann. Inst. H. Poincare 16, 63-73 (1980)

8. Grimmett, G.R., Stirzaker, D.R.: Probability and Random Processes, 3rd edn. Oxford University Press, New York (2001)

9. Keilson, J., Servi, L.: Oscillating random walk models for G/G/1 vacation systems with bernoulli schedules. J. Appl. Probab. 23, 790-802 (1986)

10. Kella, O., Taksar, M.I.: A heavy traffic limit for the cycle counting process in G/G/1, optional interruptions and elastic screen Brownian motion. Math. Oper. Res. 19, 132-151 (1994)

11. Kella, O., Whitt, W.: Queues with server vacations and Lévy processes with secondary jump input. Ann. Appl. Probab. 1, 104-117 (1991)

12. Lucantoni, D.M., Meier-Hellstern, K.S., Neuts, M.F.: A single-server queue with server vacations and a class of non-renewal arrival processes. Adv. Appl. Probab. 22, 676-705 (1990)

13. Kella, O., Whitt, W.: Diffusion approximations for queues with server vacations. Adv. Appl. Probab. 22, 706-729 (1990)

14. Shanthikumar, J.G., Sumita, U.: Modified Lindley process with replacement: dynamic behavior, asymptotic decomposition and applications. J. Appl. Probab. 26, 552-565 (1989) 